\title{
Danzhi Jiangtang capsule regulates the metabolism of blood lipids in hyperlipidemic rats through JAK2/STAT3 pathway
}

\author{
Gang YIN ${ }^{1 *}\left(\mathbb{D}\right.$, Guoming SHEN ${ }^{1}$, Tingting $\mathrm{DAI}^{2}$, Jingya $\mathrm{LI}^{2}$
}

\begin{abstract}
Danzhi Jiangtang capsule (DJC), a traditional medicinal formula composed of cortex moutan, heterophylly falsestarwort root, unprocessed rehmannia root, oriental waterplantain rhizome, dodder seed and leech, has been used to treat type 2 diabetes in clinic for almost 10 years. In addition, DJC was also confirmed that it could relieved oxidative stress of vascular endothelium in diabetic macroangiopathy. Based on the fact that the DJC could regulated the level of free fatty acids in diabetes patients and the constituents of DJC also possess potential anti-hyperlipidemic effect, thus, the present work aimed to evaluate the anti-hyperlipidemic effect of DJC and further investigated potential molecular mechanism of this action. As in vivo results revealed, DJC could reduced the levels of TC, TG, LDL, increased HDL level as well as decreased the level of ALT and AST of model rats. In addition, DJC also showed significant antioxidant effect by decreasing the level of MDA and increasing the level of SOD and GSH-Px. Furthermore, western blot analysis showed that DJC could up-regulated the expression of phosphorylated JAK2 and STAT3, and down-regulated the expression of SCOC3 protein, which indicated that DJC could regulate the metabolism of lipid via JAK2/STAT3 pathway.
\end{abstract}

Keywords: Danzhi Jiangtang capsule (DJC); anti-hyperlipidemic effect; JAK2/STAT3; rat; Lipid metabolism.

Practical Application: In this study, Danzhi Jiangtang capsule (DJC) showed the anti-hyperlipidemic effect and significant antioxidant effect and could regulate the metabolism of lipid.

\section{Introduction}

Based on the World Health Organization (WHO) report in 2014, almost 422 million people suffered from diabetes worldwide (World Health Organization, 2018). In these patients, most people were diagnosed with type 2 diabetes (T2DM), which resulted from insufficiency of insulin secretion or insulin action and the disordered metabolism of carbohydrate (van Roozendaal \& Krass, 2009). Obesity plays a key role in the development of T2DM, and be considered as one of the largest endocrine disease which caused high mortality rate. In fact, it is a chronic disease caused by imbalance between the level of energy intake and expenditure, with the surplus being stored as body lipids (Kim et al., 2014). WHO has estimated that in 2016 more than $39 \%$ of the population were overweight (1.9 billion) and 13\% were with obesity (650 million) (World Health Organization, 2018). Actually, in addition to reasonable eating habits and exercise, pharmacotherapy is currently the primary strategy to treat obesity and some drugs including bile acid sequestrants, fibrates as well as nicotinic acids have been used as anti-obesity and lipid-lowering agents. However, some considerable side effects such as gastrointestinal, liver, and muscle toxicity are often observed, limiting their utilization (Williams et al., 2020). WHO encouraged investigations to search the alternative remedies derived from traditional herbal medicines. Thus, in this paper, we investigate anti-obesity activity of Danzhi Jiangtang capsule
(DJC), a traditional Chinese medicine which be used for treating T2DM in clinic for about 10 years.

DJC is composed of the $21.6 \%$ of cortex moutan, $27.1 \%$ of heterophylly falsestarwort root, $21.6 \%$ of unprocessed rehmannia root, $16.2 \%$ of oriental waterplantain rhizome, $10.8 \%$ of dodder seed and $2.7 \%$ of leech (Zheng et al., 2016). Previous studies have reported that DJC could regulated the level of free fatty acids in diabetes patients, and the constituents of DJC also possessed potential anti-hyperlipidemic effect (Fang et al., 2020; $\mathrm{Lu}$ et al., 2018). All above stimulated us to fully investigate the anti-hyperlipidemic effect of DJC and its in-depth mechanism of this action.

\section{Materials and methods}

\subsection{Experimental animals and ethical aspects}

Wistar rats, male, weighing $180 \pm 20 \mathrm{~g}$, were purchased from Experimental Animal Center of Anhui University of Chinese Medicine, certificate number: SCXK 2019-022. All animals were housed in individual cages under a $12 \mathrm{~h} \mathrm{light} / 12 \mathrm{~h}$ dark cycle and controlled temperature $\left(25 \pm 2{ }^{\circ} \mathrm{C}\right)$ and relative humidity of $40-60 \%$. The protocol for this study involving animals and their maintenance was approved by Animal Care and Use 
Committee of Anhui University of Chinese Medicine (Approved no. AUCM-301-05-02).

\subsection{Preparation of high-fat emulsion}

The high-fat emulsion was prepared as previously reported. (Aslan et al., 2010) Briefly, $25 \mathrm{~g}$ of lard oil in a $200 \mathrm{~mL}$ beaker were heated on a magnetic stirring apparatus. When the temperature reached $100^{\circ} \mathrm{C}, 10 \mathrm{~g}$ of cholesterol were added and melted. The oil phase was prepared by stirring $1 \mathrm{~g}$ of methylthiouracil into the mixture followed by the addition of $25 \mathrm{~mL}$ of tween- 80 . Simultaneously, in another $200 \mathrm{~mL}$ beaker, $30 \mathrm{~mL}$ of distilled water and $20 \mathrm{~mL}$ of propylene glycol were mixed and heated using an electric oven. When the temperature reached $60{ }^{\circ} \mathrm{C}$, 2 grams of sodium deoxycholate were added, and the mixture was stirred until all components were dissolved. Subsequently, the water phase prepared in this beaker was added to the oil phase and mixed thoroughly to prepare the high-fat emulsion.

\subsection{Animals groups and models}

Sixty rats were acclimatized for 3 days with free access to water and normal pellet, and were randomly divided into 2 groups. In the first stage, the normal group $(\mathrm{n}=10)$ was given intragastric $0.9 \%$ saline, $10 \mathrm{~mL} / \mathrm{kg}$, while the other groups (n $=50$ ) were given the amount of the high fat emulsion in the morning (intragastric administration of $10 \mathrm{~mL} \cdot \mathrm{kg}^{-1}$ ). After 28 days, blood samples were collected from caudal vein and the serum lipid level was measured. There was a significant difference in serum level of TC and TG between normal group and hyperlipidaemia model group, suggesting the hyperlipidemia model has been established. In the second stage, each group, except the control group, were given high fat emulsion in the morning. The control group was given the same amount of $0.9 \%$ saline. These hyperlipidemic animals were then randomly divided into 5 groups including the model, simvastatin, low-, medium- and high dose of DJC groups. Every afternoon, the simvastatin group was administered with simvastatin solution at a dose of $5 \mathrm{mg} / \mathrm{kg}$ body weight/day. The low-, medium- and high dose groups were administered with the DJC at a dose of 250,500 and $1000 \mathrm{mg} / \mathrm{kg}$ body weight/day, respectively. This stage of administration lasted for 4 weeks. The rats were allowed for free access to water and food during the whole experiment. And the body weight and food consumption were measured once a week.

\subsection{Measurement of indices}

All rats were weighed weekly in the period of the whole experiment. Blood samples were collected, then immediately centrifuged at $2500 \mathrm{rpm}$ for $20 \mathrm{~min}$ to obtain the serum. The content of TC, TG, LDL, HDL, ALT, AST SOD, MDA and GSH-Px in serum were determined with commercial kits (Jiancheng Biological Company, Nanjing, China).

\subsection{Western blot analysis}

The liver tissue was collected after rat in each group were sacrificed and further western blot analysis was performed using the established methods. (Lee et al., 2006) Briefly, liver tissues were weighed, lysed with RIPA lysis buffer containing protease and phosphatase inhibitor cocktails (Solarbio, Beijing, China) for $20 \mathrm{~min}$, and then homogenized on the ice to extract the protein. The protein concentrations were quantified using the Protein Assay Kit (Beyotime, Shanghai, China). Samples were subjected to $10 \%$ SDS-polyacrylamide gel electrophoresis (SDSPAGE) then transferred onto polyvinylidene fluoride (PVDF) membranes. After blocking PVDF membranes with 5\% nonfat powdered milk, membranes were washed three times using TBST (50 m MTris/HCl pH 7.4, $150 \mathrm{mM} \mathrm{NaCl}, 0.1 \%$ Tween 20 ) and each time was 5 mins, incubated with specific primary antibodies (dilution of 1:1000) against phospho-JAK2, JAK2, phospho-STAT3, STAT3, SOCS- 3 at $4{ }^{\circ} \mathrm{C}$ overnight. Next day membranes were washed with TBST three times and each time was 5 mins. Then membranes were exposed to secondary antibody (dilution of 1:5000) for $1 \mathrm{~h}$, washed three times again with TBST and each time was 10 mins, stained using the ECL Western Blotting Kit according to the manufacturer's specifications. Eventually, films were took and the light density of target bar was analyzed by ImageJ software. $\beta$-actin (1:1000 dilution) was used as a loading control.

\subsection{Statistical analysis}

All presented results were analyzed and calculated by at least three independent experiments and these data were expressed as means $\pm S D$. One-way ANOVA was used to compare quantitative data among groups through GraphPad Prism Software. LSD-post hoc test was employed to evaluate the statistical significance of difference between control and treated groups. $p<0.05$ was considered to indicate a statistically significant difference.

\section{Results and discussion}

In order to evaluate the reliability of model group, 3 rats from the control and model groups were respectively picked after 28 days and the changes of the level of TC and TG were tested. As shown in Table 1, compared with that of control group, the levels of TC and TG in model group rats were increased substantially $(P<0.01)$. In addition, All rats of being given high-fat emulsion were heavier than normal group throughout this experiment (Table 2). However, there was no striking difference in weight between the different treated groups. The above data indicated that the establishment of model group was successful.

At the end stage of this experiment, rats were fasted for $12 \mathrm{~h}$ after the last dose. On the $28^{\text {th }}$ day, blood samples were collected from each rat and the content of total cholesterol (TC), triglycerides (TG), lipoprotein (LDL), high-density lipoprotein (HDL), alanine aminotransferase (ALT), aspartate aminotransferase (AST), superoxide dismutases (SOD), malondialdehyde (MDA)

Table 1. Serum lipids of rats in normal and model groups.

\begin{tabular}{cccc}
\hline Group & $n$ & TC $(\mathrm{mmol} / \mathrm{L})$ & TG $(\mathrm{mmol} / \mathrm{L})$ \\
\hline Normal group & 3 & $2.01 \pm 0.35$ & $0.68 \pm 0.08$ \\
Model group & 3 & $6.29 \pm 0.56^{*}$ & $0.82 \pm 0.10^{*}$ \\
\hline${ }^{*} P<0.01$ compared with the normal group. & &
\end{tabular}

${ }^{\star} P<0.01$ compared with the normal group. 
and glutathione peroxidase (GSH-Px) were all determined by the automatic biochemistry analyser. Compared with the model group, there is a significant decrease in the levels of serum TC, TG and LDL and a obvious increase in the level of HDL in three treated groups, though they did not return to normal values $(P$ $<0.05$ ). In addition, the high dose group showed more potent effect than low and medium-dose groups $(P<0.05)$, indicating that DJC exerted its potential anti-hyperlipidemic effect in a dose dependent manner (Table 3).

MDA is a product of the oxidation of unsaturated fatty acid induced by free radicals, the contents of which could indirectly reflect the extent of oxidation of body fat (Liu \& $\mathrm{Ng}, 2000$ ). $\mathrm{SOD}$ is the main enzyme to remove $\mathrm{O}^{2}$ the body. It can catalyze disproportionating reaction of $\mathrm{O}^{2}$ - as $\mathrm{H}_{2}$ and $\mathrm{O}^{2}$, which plays an important role in keeping the balance of oxidation and antioxidant in the body. GSH-Px, is a kind of essential antioxidant, which can remove the free oxygen radicals produced by the metabolism in physiological states and protecting cells. In this study, compared with model group, all three treated groups remarkably decreased the active levels of MDA $(P<0.01)$, while the high dose group significantly increased the levels of SOD and GSH-Px $(P<0.01)$.
The results of this study revealed that high dose group might could remove free radicals to protect the liver (Table 4).

The liver plays a critical role in the normal metabolism of energy substrates, particularly lipid metabolism. AST and ALT are the main indicators for evaluating the response of liver injury and the liver function (Umar et al., 2015). And this two enzymes mainly exist in the liver cells and mitochondria and cytosol, respectively. When the liver is compromised, AST concentrations in serum increase significantly (Hu et al., 2011). Concentrations of serum ALT and AST rise significantly, indicating liver damage in hyperlipaemic rats. In this experiment, compared with the normal group, simvastatin and other three treated groups decreased the levels of ALT and AST, indicating that DJC could restore liver function in a certain extent. However, the effect of three treated groups on ALT and AST was not noticeably greater than that of simvastatin (Table 5).

The possible mechanism of regulating lipid metabolism of DJC was also explored herein. Leptin are responsible for controlling the food intake, thermogenesis and energy consumption of body (Morrison et al., 2014). Leptin resistance,

Table 2. Effect of DJC extract on body weight of rats during 4 weeks (Mean $\pm \mathrm{SD}, n=10$ ).

\begin{tabular}{cclllll}
\hline Groups & Dose $(\mathrm{mg} / \mathrm{kg})$ & \multicolumn{1}{c}{0 week $(\mathrm{g})$} & \multicolumn{1}{c}{1 week $(\mathrm{g})$} & 2 weeks $(\mathrm{g})$ & 3 weeks $(\mathrm{g})$ & 4 weeks $(\mathrm{g})$ \\
\hline Normal group & - & $202.02 \pm 10.03$ & $220.21 \pm 13.72$ & $234.60 \pm 13.21$ & $241.29 \pm 14.84$ & $247.30 \pm 9.89$ \\
Model group & - & $227.14 \pm 5.67$ & $248.12 \pm 9.64$ & $270.08 \pm 12.58$ & $280.58 \pm 14.74$ & $287.10 \pm 14.21$ \\
Simvastatin & 5 & $198.45 \pm 10.93$ & $226.16 \pm 10.03$ & $240.08 \pm 16.31$ & $251.31 \pm 13.90$ & $260.15 \pm 17.32$ \\
DJC-L & 250 & $205.83 \pm 15.26$ & $237.40 \pm 15.35$ & $258.62 \pm 14.41$ & $275.08 \pm 9.60$ & $282.70 \pm 18.24$ \\
DJC-M & 500 & $197.26 \pm 13.30$ & $238.40 \pm 12.17$ & $254.50 \pm 13.86$ & $263.17 \pm 12.10$ & $272.40 \pm 18.80$ \\
DJC-H & 1000 & $201.27 \pm 15.08$ & $233.60 \pm 14.15$ & $250.20 \pm 17.34$ & $255.40 \pm 16.37$ & $264.30 \pm 18.70$ \\
\hline
\end{tabular}

DJC-L: low dosage of Danzhi Jiangtang capsule. DJC-M: medium dosage of Danzhi Jiangtang capsule. DJC-H: high dosage of Danzhi Jiangtang capsule. Significant statistical difference was not observed between the weights of each group of rats $(P>0.05)$.

Table 3. Effects of DJC on the levels of TC, TG, LDL-C, and HDL-C of rats (Mean \pm SD, $n=10$ ).

\begin{tabular}{cccccc}
\hline Groups & Dose $(\mathrm{mg} / \mathrm{kg})$ & TC $(\mathrm{mmol} / \mathrm{L})$ & TG $(\mathrm{mmol} / \mathrm{L})$ & LDL-C $(\mathrm{mmol} / \mathrm{L})$ & HDL-C $(\mathrm{mmol} / \mathrm{L})$ \\
\hline Normal group & - & $2.14 \pm 0.32$ & $0.50 \pm 0.10$ & $0.48 \pm 0.17$ & $4.05 \pm 0.36$ \\
Model group & - & $6.17 \pm 1.11^{\star}$ & $0.88 \pm 0.07^{*}$ & $5.29 \pm 2.10^{* *}$ & $2.42 \pm 0.19^{* *}$ \\
Simvastatin & 5 & $4.07 \pm 0.43^{\star}$ & $0.41 \pm 0.07^{\star}$ & $4.26 \pm 1.79$ & $4.08 \pm 0.61^{* *}$ \\
DJC-L & 250 & $5.68 \pm 1.21$ & $0.64 \pm 0.09^{* *}$ & $5.29 \pm 1.75$ & $3.90 \pm 0.31^{* *}$ \\
DJC-M & 500 & $5.01 \pm 1.28^{* *}$ & $0.50 \pm 0.08^{*}$ & $4.71 \pm 1.87^{* *}$ & $4.10 \pm 0.33^{* *}$ \\
DJC-H & 1000 & $4.21 \pm 0.38^{* *}$ & $0.44 \pm 0.05^{* *}$ & $4.50 \pm 0.89^{* *}$ & $4.01 \pm 0.39^{* *}$
\end{tabular}

DJC-L: low dosage of Danzhi Jiangtang capsule. DJC-M: medium dosage of Danzhi Jiangtang capsule. DJC-H: high dosage of Danzhi Jiangtang capsule. ${ }^{\star} P<0.05$ compared with the hyperlipidaemia model group; ${ }^{* *} P<0.01$ compared with the hyperlipidaemia model group.

Table 4. Effect of DJC on rat serum SOD, MDA and GSH-Px (Mean \pm SD, $n=10)$.

\begin{tabular}{|c|c|c|c|c|}
\hline Groups & Dose $(\mathrm{mg} / \mathrm{kg})$ & $\mathrm{SOD}(\mathrm{U} / \mathrm{L})$ & $\mathrm{MDA}(\mathrm{nmol} / \mathrm{L})$ & GSH-Px (pmol/L) \\
\hline Normal group & - & $226.82 \pm 12.43$ & $4.51 \pm 0.47$ & $43.31 \pm 3.08$ \\
\hline Model group & - & $192.17 \pm 11.47$ & $6.90 \pm 1.12^{\star}$ & $35.49 \pm 1.10$ \\
\hline Simvastatin & 5 & $211.39 \pm 8.76^{*}$ & $4.42 \pm 0.51$ & $39.50 \pm 2.14^{\star}$ \\
\hline DJC-L & 250 & $201.71 \pm 9.20^{* *}$ & $4.40 \pm 0.38^{\star}$ & $37.47 \pm 1.48^{\star *}$ \\
\hline DJC-M & 500 & $207.76 \pm 8.49$ & $3.91 \pm 0.43^{\star *}$ & $38.43 \pm 1.79^{*}$ \\
\hline DJC-H & 1000 & $220.18 \pm 6.79^{*}$ & $3.72 \pm 0.65^{\star *}$ & $42.42 \pm 1.13^{\star *}$ \\
\hline
\end{tabular}

DJC-L: low dosage of Danzhi Jiangtang capsule. DJC-M: medium dosage of Danzhi Jiangtang capsule. DJC-H: high dosage of Danzhi Jiangtang capsule. ${ }^{\star} P<0.05$ compared with the hyperlipidaemia model group; ${ }^{* *} P<0.01$ compared with the hyperlipidaemia model group. 
Table 5. Effect of DJC on the level of serum AST, ALT and ALT/AST (Mean \pm SD, $n=10$ ).

\begin{tabular}{ccccc}
\hline Groups & Dose $(\mathrm{mg} / \mathrm{kg})$ & AST $(\mathrm{U} / \mathrm{L})$ & ALT $(\mathrm{U} / \mathrm{L})$ & ALT/AST \\
\hline Normal group & - & $90.60 \pm 8.23$ & $35.56 \pm 4.67$ & $0.41 \pm 0.08$ \\
Model group & - & $125.30 \pm 10.38$ & $62.40 \pm 8.08$ & $0.49 \pm 0.10$ \\
Simvastatin & 5 & $98.11 \pm 7.92^{*}$ & $51.00 \pm 11.21$ & $0.50 \pm 0.14$ \\
DJC-L & 250 & $120.08 \pm 9.38^{* *}$ & $57.08 \pm 4.38^{* *}$ & $0.47 \pm 0.08^{\star *}$ \\
DJC-M & 500 & $118.93 \pm 7.64$ & $51.02 \pm 7.41^{\star}$ & $0.43 \pm 0.09^{\star}$ \\
DJC-H & 1000 & $110.22 \pm 4.15^{*}$ & $46.42 \pm 5.93^{* *}$ & $0.42 \pm 0.10^{* *}$ \\
\hline
\end{tabular}

DJC-L: low dosage of Danzhi Jiangtang capsule. DJC-M: medium dosage of Danzhi Jiangtang capsule. DJC-H: high dosage of Danzhi Jiangtang capsule. ${ }^{*} P<0.05$ compared with the hyperlipidaemia model group; ${ }^{* *} P<0.01$ compared with the hyperlipidaemia model group.

$\mathbf{A}$

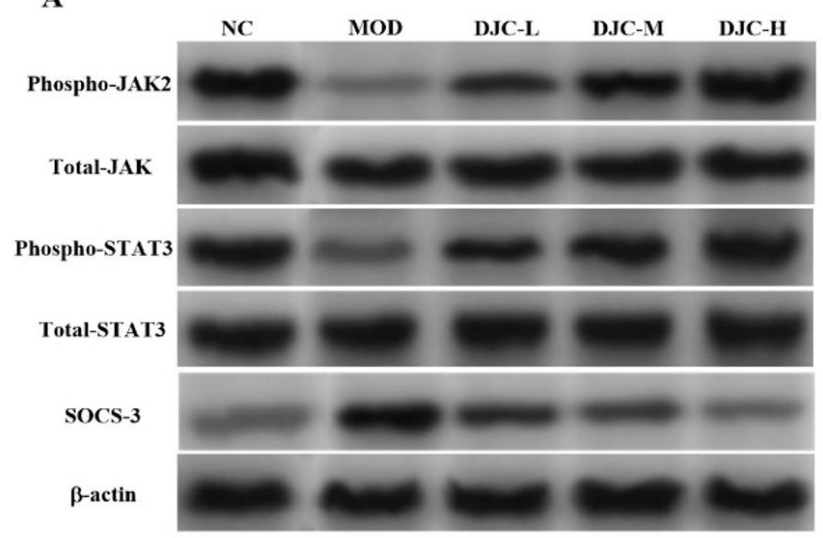

B

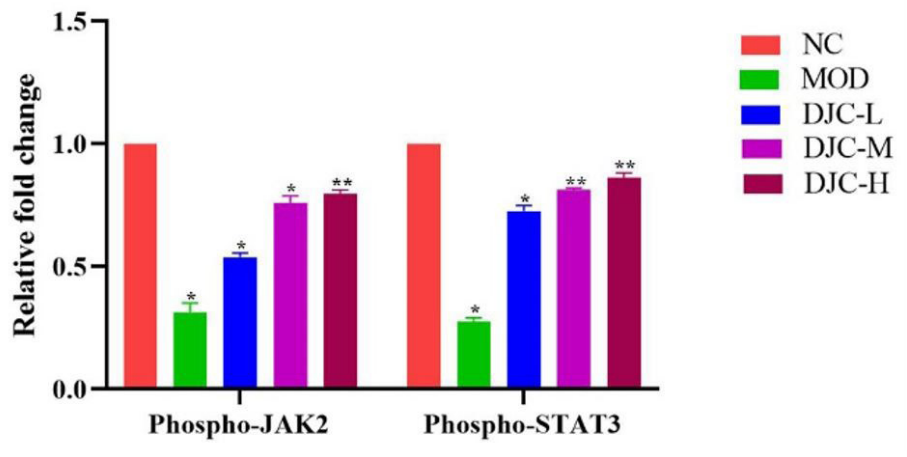

Figure 1. The effect of JAK2/STAT3 pathways in the liver. (A) The changes of expressions of $p$-JAK2, JAK2, p-STAT3, STAT3 and SOCS-3 proteins. (B) The changes of relative densities of proteins by densitometric analysis of blots using Image J software. ${ }^{\star} p<0.05 ;{ }^{\star *} p<0.01$.

defined as an impaired neuronal response to leptin, is involved in the occurrence of obesity. The up-regulated level of leptin was commonly observed in the most obese individuals. JAK2/ STAT3 pathway is the main signal regulated by leptin, and appropriate expressions of JAK2, STAT3 and SOCS-3 proteins are important for maintaining the normal hepatic function (Chen et al., 2019). However, the abnormal expressions of JAK2, STAT3, and SOCS-3 will lead to the accumulation of fat in the liver and even fatty liver. In this study, western blot analysis showed that DJC could significantly up-regulated the expression of phosphorylated JAK2 and STAT3, and down-regulated the level of SOCS-3 expression in a dose dependent manner. These results indicated that DJC could decreased the lipid metabolism via JAK2/STAT3 pathway (Figure 1).

\section{Conclusion}

In conclusion, this study first demonstrated that DJC could reduced the levels of TC, TG, LDL, increased HDL level as well as displayed the potential protective effect through decreasing the expression of ALT and AST of model rats. And DJC also showed antioxidant effect by decreasing the level of MDA and increasing the level of SOD and GSH-Px. Furthermore, western blot analysis showed that DJC could up-regulated the expression of phosphorylated JAK2 and STAT3, and down-regulated the expression of SCOC3 protein to regulate the lipid metabolism in hyperlipidaemia model rats.

\section{Conflict of interest disclosure}

The authors declare no conflict of interest regarding the publication of this manuscript.Statement of Informed Consent.

\section{Funding}

This study was supported by General Projects of Anhui Natural Science Foundation (1708085MH201).

\section{Author contributions}

GY designed the experiments and examined the manuscript. GGS and TTD carried out the experiment. GGS and YJL wrote the manuscript and supervised the research. All authors discussed the results and contributed to the final manuscript.

\section{Acknowledgments}

The authors are thankful for the help from the colleagues of Department of Anhui University of Chinese Medicine.

\section{References}

Aslan, M., Orhan, N., Orhan, D. D., \& Ergun, F. (2010). Hypoglycemic activity and antioxidant potential of some medicinal plants traditionally used in Turkey for diabetes. Journal of Ethnopharmacology, 128(2), 384-389. http://dx.doi.org/10.1016/j.jep.2010.01.040. PMid:20100559. 
Chen, Y., Lu, W., Jin, Z., Yu, J., \& Shi, B. (2019). Carbenoxolone ameliorates hepatic lipid metabolism and inflflammation in obese mice induced by high fat diet via regulating the JAK2/STAT3 signaling pathway. International Immunopharmacology, 74, 105498. http://dx.doi. org/10.1016/j.intimp.2019.03.011. PMid:31261036.

Fang, Z., Hu, X., Chen, Z., Xie, J., Wu, D., Yin, Y., \& You, L. (2020). Radix pseudostellariae of Danzhi Jiangtang capsule relieves oxidative stress of vascular endothelium in diabetic macroangiopathy. Saudi Pharmaceutical Journal, 28(6), 683-691. http://dx.doi.org/10.1016/j. jsps.2020.04.009. PMid:32550799.

Hu, Y. J., Zhang, J. C., Wang, L., \& Cheng, L. (2011). Regulation of lipid metabolism and vascular endothelial function by soft capsule of yindan xinnaotong in hyperlipidemia rats. Zhongguo Shiyan Fangjixue Zazhi, 21, 162-164. http://dx.doi.org/10.3969/j.issn.10059903.2011.21.045.

Kim, S. B., Ahn, B., Kim, M., Ji, H. J., Shin, S. K., Hong, I. P., Kim, C. Y., Hwang, B. Y., \& Lee, M. K. (2014). Effect of Cordyceps militaris extract and active constituents on metabolic parameters of obesity induced by high-fat diet in C58BL/6J mice. Journal of Ethnopharmacology, 151(1), 478-484. http://dx.doi.org/10.1016/j. jep.2013.10.064. PMid:24231073.

Lee, S. C., Chan, J., Clement, M. V., \& Pervaiz, S. (2006). Functional proteomics of resveratrol-induced colon cancer cell apoptosis: caspase-6-mediated cleavage of lamin A is a major signaling loop. Proteomics, 6(8), 2386-2394. http://dx.doi.org/10.1002/pmic.200500366. PMid:16518869.

Liu, F., \& Ng, T. B. (2000). Antioxidative and free radical scavenging activities of selected medicinal herbs. Life Sciences, 66(8), 725-735. http://dx.doi.org/10.1016/s0024-3205(99)00643-8. PMid:10680580.

Lu, Y., Chen, Y., Li, R., Liu, Q., Wang, N., Zhang, Y., Li, B., \& Fang, Z. (2018). Protective effects of Danzhi jiangtang capsule on vascular endothelial damages induced by high-fat diet and palmitic acid. Biomedicine and Pharmacotherapy, 107, 1631-1640. http://dx.doi. org/10.1016/j.biopha.2018.08.129. PMid:30257381.

Morrison, S. F., Madden, C. J., \& Tupone, D. (2014). Central neural regulation of brown adipose tissue thermogenesis and energy expenditure. Cell Metabolism, 19(5), 741-756. http://dx.doi. org/10.1016/j.cmet.2014.02.007. PMid:24630813.

Umar, A., Iskandar, G., Aikemu, A., Yiming, W., Zhou, W. T., Berke, B., Begaud, B., \& Moore, N. (2015). Effects of Cydonia oblonga Miller leaf and fruit flavonoids on blood lipids and anti-oxydant potential in hyperlipidemia rats. Journal of Ethnopharmacology, 169(1), 239243. http://dx.doi.org/10.1016/j.jep.2015.04.038. PMid:25934516.

van Roozendaal, B. W., \& Krass, I. (2009). Development of an evidencebased checklist for the detection of drug related problems in type 2 diabetes. Pharmacy World \& Science, 31(5), 580-595. http://dx.doi. org/10.1007/s11096-009-9312-1. PMid:19626455.

Williams, D. M., Nawaz, A., \& Evans, M. (2020). Drug therapy in obesity: A review of current and emerging treatments. Diabetes Therapy: Research, Treatment and Education of Diabetes and Related Disorders, 11(6), 1199-1216. http://dx.doi.org/10.1007/s13300-02000816-y. PMid:32297119.

World Health Organization - WHO. (2018). Fact sheet-obesity and overweight. Retrieved from http://www.who. int/news-room/factsheet s/detai 1/obesi ty-and-overw

Zheng, S., Zhao, M., Wu, Y., Wang, Z., \& Ren, Y. (2016). Suppression of pancreatic beta cell apoptosis by Danzhi Jiangtang capsule contributes to the attenuation of type 1 diabetes in rats. BMC Complementary and Alternative Medicine, 16, 31. http://dx.doi.org/10.1186/s12906016-0993-4. PMid:26819084. 